\title{
An Unusual Localization of the Umbilicus in a Neonate
}

\author{
Alkim Öden Akman ${ }^{1} \quad$ Nazile Erturk $^{2}$ Sumru Kavurt ${ }^{1}$ \\ Ahmet Yagmur Bas ${ }^{1}$ Nihal Demirel ${ }^{1}$
}

Ulker Celik $^{1}$ Ozge Aydemir ${ }^{1} \quad$ Cuneyt Yesiltepe $^{3}$

Address for correspondence Sumru Kavurt, MD, Department of Neonatology, Etlik Zübeyde Hanim Women's Health Teaching and Research Hospital, Yeni Etlik Caddesi 55, Ankara Etlik 06010, Turkey (e-mail: sumrukavurt@gmail.com).

\author{
Abstract \\ Keywords \\ - low-set umbilicus \\ - neonate \\ - umbilical cord
}

We report the case of a male neonate with a low-set umbilicus. Physical examination revealed an appropriately grown term infant with no unusual findings, except ectopically placed umbilical cord at the level of the bladder in the hypogastric zone. The infant underwent detailed investigations that revealed no associated malformation. To the best of our knowledge, this is the first case with very distinct localization of the umbilicus without any congenital abnormalities.

\section{Introduction}

The umbilicus is a round dermal projection on the center of anterior abdominal wall, which is at the level of the fibrocartilage between the third and fourth lumbar vertebrae. ${ }^{1}$ There are few articles published investigating the normal localization of the umbilicus in the newborn population to guide pediatric surgeon for a new umbilical position after repair of certain congenital defects. ${ }^{2,3}$ Little is known about the cause of inferior displacement of the umbilical cord, accompanying symptoms and any related clinical outcomes.

We report a case with very distinct localization of the umbilicus without any congenital abnormalities.

\section{Case Report}

A boy, weighing $3,210 \mathrm{~g}$ (50th to 75 th percentile), with a head circumference of $36 \mathrm{~cm}$ (50th to 75th percentile) and a height of $51 \mathrm{~cm}$ (50th to 75th percentile) was born at 38 weeks of gestation by vaginal delivery.

The pregnancy was uncomplicated. Apgar scores were 8 and 9 at 1 and 5 minutes, respectively, and no resuscitation was required. Parents were third degree relatives, and one of his sisters died at an age of 5.5 months due to complications after myelomeningocele surgery. Rest of the family history was unremarkable.

Physical examination revealed an appropriately grown term infant with no unusual findings except ectopically placed umbilical cord just above the symphysis pubis, $1.1 \mathrm{~cm}$ to the root of the penis ( - Fig. 1). The diameter and circumference of the umbilical cord were 1.6 and $5 \mathrm{~cm}$, respectively. The umbilical cord had two arteries and one vein, which were surrounded by Wharton jelly. Distances between the xiphoid process and the center of the umbilicus $(\mathrm{XU})$, the pubis and the center of the umbilicus (PU), and the xiphoid process and pubis (XP) were 11.4, 0.6, and $12 \mathrm{~cm}$, respectively. The PU:XU ratio was calculated as 0.05 .

Results of laboratory evaluation, including serum electrolytes, blood urea nitrogen, serum creatinine tests were unrevealing. The chest radiograph shows a normal heart size and normal lung fields with no consolidation. The abdominal radiograph shows nonspecific bowel-gas with no evidence of obstruction or free air. The patient had normal skeletal survey. Abdominal ultrasound scanning, including the bladder wall and the other abdominal organs, voiding cystourethrogram, cranial ultrasound, and echocardiogram demonstrated normal anatomy. Transcutaneous Doppler ultrasound measurements received

November 9, 2012

accepted

November 22, 2012

published online

March 8, 2013 (c) 2013 Georg Thieme Verlag KG Stuttgart · New York
DOI http://dx.doi.org/ 10.1055/s-0033-1337113. ISSN 2194-7619. 


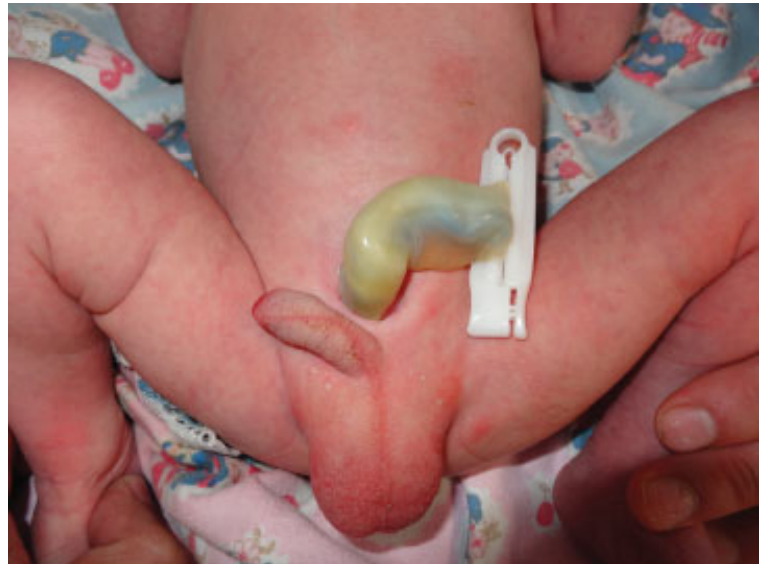

Fig. 1 Low-set umbilicus.

of iliac and mesenteric artery blood flow were performed and revealed normal flow velocities.

The umbilical cord fell off within the 10 days with an uneventful clinical course. Soft tissue around umbilicus seemed normal without any signs of infection.

\section{Discussion}

The umbilical cord develops from and contains remnants of the yolk sac and allantois. It forms by the fifth week of fetal development, replacing the yolk sac as the source of nutrients for the fetus. An average umbilical cord is $55 \mathrm{~cm}$ long, with a diameter of 1.5 to $3.6 \mathrm{~cm} .{ }^{4,5}$ The anatomical text describes the umbilicus as being at the level of the fibrocartilage between the third and fourth lumbar vertebrae. ${ }^{6}$

The position of the umbilical cord is established at the time of umbilical ring formation. It is located at the junction of four body folds including the cranial, the caudal and two lateral folds that comprise anterior body wall. A high, low, or eccentric position of the umbilicus on the surface of the anterior abdominal wall is considered an abnormality of umbilical cord position. The eccentric umbilical cord or umbilicus is located away from midline. High or low cords are usually located on the midline. Abnormalities related to an excess of mesodermal derivatives from the septum transversum may result in inferior displacement of the umbilical cord position. ${ }^{7}$

Knowledge about normal variations in umbilicus locations can help in diagnosis of pathologies of abdominal wall development and determination of some syndromes. Few studies evaluated normal localization of the umbilicus in the newborn, which aid to improve cosmetic results after the repair of congenital abdominal wall defects. ${ }^{2,3,6}$ These studies determined the position of umbilicus with respect to the xiphoid process and pubis in normal newborns. Distances between the xiphoid process and the center of the umbilicus (XU), the pubis and the center of the umbilicus (PU) were determined in a study. The PU:XU ratio for an ideal localization of umbilicus was calculated as $0.61 \pm 0.12$ in this study. ${ }^{2} \mathrm{PU}: \mathrm{XU}$ ratio was 0.05 in our patient, which is approximately one-tenth of the ratio for ideal localization.

Low-set umbilicus may be a finding in disorders such as renal agenesis-dysplasia, single umbilical artery, fetal growth retardation, hydrops fetalis, monozygous twinning, anencephaly, achondroplasia, and Robinow syndrome. ${ }^{1,7}$

Physical examination of the present case revealed no dysmorphic features. He underwent a detailed investigation that included sonographic examinations, echocardiography, voiding cystourethrogram and skeletal survey. Associated malformation was not detected.

To conclude, specific treatment of abnormal umbilical position in our patient is not indicated because it is not a life-threating event. However, surgical intervention may be considered for cosmetic reasons. To the best of our knowledge, this is the first case with very distinct localization of the umbilicus without any congenital abnormalities.

\section{Conflict of Interest}

None

\section{References}

1 Coetzee T. Clinical anatomy of the umbilicus. S Afr Med J 1980; 57(12):463-466

2 Onal E, Turan O, Karabulut R, et al. Where should the normal position of the umbilicus be in the neonate? Eur J Pediatr Surg 2010;20(5):339-340

3 Williams AM, Brain JL. The normal position of the umbilicus in the newborn: an aid to improving the cosmetic result in exomphalos major. J Pediatr Surg 2001;36(7):1045-1046

4 Moinian M, Meyer WW, Lind J. Diameters of umbilical cord vessels and the weight of the cord in relation to clamping time. Am J Obstet Gynecol 1969;105(4):604-611

5 Patel D, Dawson M, Kalyanam P, et al. Umbilical cord circumference at birth. Am J Dis Child 1989;143(6):638-639

6 Davari HA, Nazem M, Hosseinpour M. The normal position of the umbilicus in the newborn: An aid to improving the cosmetic result in exomphalos major. J Ind Assoc Pediatr Surg. 2006;11: 133-135

7 Blackburn W. The umbilical cord. In: Stevenson RE, Hall JG, Goodman RM, eds. Human Malformations and Related Anomalies. 2nd ed. New York, NY: Oxford University Press; 2006: $1413-1472$ 